\title{
The substellar mass function in the central region of the open cluster Praesepe from deep LBT observations ${ }^{\star}$
}

\author{
W. Wang ${ }^{1,2}$, S. Boudreault ${ }^{1,3,4}$, B. Goldman ${ }^{1}$, Th. Henning ${ }^{1}$, J. A. Caballero ${ }^{5}$, and C. A. L. Bailer-Jones ${ }^{1}$ \\ ${ }^{1}$ Max-Planck-Institut für Astronomie, Königstuhl 17, 69117 Heidelberg, Germany \\ e-mail: wangw@nao.cas.cn \\ 2 Key Laboratory of Optical Astronomy, National Astronomical Observatories, Chinese Academy of Sciences, \\ Beijing 100012, PR China \\ 3 Visiting Astronomer at the Department of Physics and Astronomy, State University of New York, Stony Brook, \\ NY 11794-3800, USA \\ ${ }^{4}$ Mullard Space Science Laboratory, University College London, Holmbury St Mary, Dorking, Surrey, RH5 6NT, UK \\ 5 Centro de Astrobiología (CSIC-INTA), Departamento de Astrofísica, PO Box 78, 28691 Villanueva de la Cañada, Madrid, Spain
}

Received 16 August 2010 / Accepted 2 May 2011

\begin{abstract}
Context. Studies of the mass function (MF) of open clusters of different ages allow us to probe the efficiency with which brown dwarfs evaporate from clusters to populate the field. Surveys of older clusters (age $\gtrsim 100 \mathrm{Myr}$ ) are not affected so severely by several problems encountered in young clusters, such as intra-cluster extinction and large uncertainties in brown dwarf models.

Aims. We present the results of a deep photometric survey to study the MF of the central region of the old open cluster Praesepe (age $590_{-120}^{+150}$ Myr, distance $\left.190_{-5.8}^{+6.0} \mathrm{pc}\right)$, down to the substellar regime.

Methods. We performed an optical (riz and $Y$-band) photometric survey of Praesepe using the Large Binocular Telescope Camera covering an area of $0.59 \mathrm{deg}^{2}$ in the cluster centre from $i \sim 19.0$ mag $\left(\sim 100 M_{\text {Jup }}\right)$ down to a $5 \sigma$ detection limit at $i \sim 25.6$ mag $\left(\sim 40 M_{\text {Jup }}\right)$. The survey is approximately $95 \%$ complete at $i=23.8 \mathrm{mag}$ and $z=22.0 \mathrm{mag}\left(\sim 55 M_{\text {Jup }}\right)$.

Results. We identify 59 cluster member candidates, of which 37 are substellar, by comparing with the predictions of a dusty atmosphere model. The MF of those candidates rises from the substellar boundary until $\sim 67 M_{\text {Jup }}$ and then declines. This is quite different from the form inferred for other open clusters older than $50 \mathrm{Myr}$, but seems to be similar to those found in very young open clusters, the MFs of which peak at $\sim 10 M_{\text {Jup }}$. Either Praesepe really does have a different MF from other clusters or they had similar initial MFs but a different dynamical evolution. Since most of the candidates are faint, we lack astrometric or spectroscopic follow-ups to test their memberships. However, the contaminations by field dwarfs, galaxies, or giants are found to have little effect on the shape of MF and therefore the MF of "real" cluster members should have similar characteristics.
\end{abstract}

Key words. brown dwarfs - stars: low-mass - stars: luminosity function, mass function - open clusters and associations: individual: Praesepe

\section{Introduction}

The mass functions (MFs) of stellar and substellar populations have been determined from optical and near-infrared surveys for several open clusters at different ages, such as the Orion Nebula Cluster, $\sigma$ Orionis, $\rho$ Ophiuchi, Taurus, IC 348, IC 2391, M 35 , the Pleiades, and the Hyades. These MFs show clear heterogeneity (see Fig. 10), which may be partially caused by cluster evolution.

Studies of relatively old open clusters (age $>100 \mathrm{Myr}$ ) are important for two particular reasons: first, they allow us to study the intrinsic evolution of basic properties of brown dwarfs (BDs), e.g., luminosity and effective temperature, and to compare the evolution with structural and atmospheric models; second, we may investigate how the BD and low-mass star populations as a whole evolve, e.g., the efficiency with which BDs and low-mass stars evaporate from clusters. These investigations have been carried out for the Hyades (Bouvier et al. 2008 and references therein) and for Praesepe (Boudreault et al. 2010 and references therein).

\footnotetext{
$\star$ Tables 2 and 3 are available in electronic form at
} http://www . aanda.org
The Praesepe open cluster has been surveyed extensively in the past (cf. Table 1), but only a few surveys have reached masses below the substellar limit (and then only just). Several BD candidates were detected in those surveys, some of which will be re-examined in the present work. The substellar MF of Praesepe remains uncertain.

Boudreault et al. (2010) observed a significant difference between the MFs of Praesepe and Hyades. While they found that the Hyades MF has a maximum at $\sim 0.6 M_{\odot}$ (Bouvier et al. 2008), the MF of Praesepe continues to rise from $0.8 M_{\odot}$ down to $0.1 M_{\odot}$. This is surprising, as both clusters share similar physical properties (ages, mass, metallicity, and tidal radii). Disagreement between the Praesepe and Hyades MFs could arise from variations in the clusters' initial MFs, or from differences in their dynamical evolution (Bastian et al. 2010). Although different binary fractions could cause the observed (system) MFs to differ, there is no clear evidence of any variations in the binary fractions from measurements published in the literature (Boudreault et al. 2010).

In this paper, we present a survey of the very low-mass star and substellar populations of Praesepe using the blue and red Large Binocular Cameras, extending down to hitherto 
Table 1. Summary of previous photometric surveys in the Praesepe around the hydrogen-burning limit.

\begin{tabular}{|c|c|c|c|c|}
\hline Authors & Telescope/instrument & $\begin{array}{l}\text { Area } \\
\left(\operatorname{deg}^{2}\right)\end{array}$ & $\begin{array}{l}\text { BD } \\
\text { candidates }\end{array}$ & $\begin{array}{l}\text { Completeness limits } \\
\text { (mag) }\end{array}$ \\
\hline Hambly et al. (1995) & COSMOS/POS \& UKSTU & 19 & 0 & $R_{\mathrm{F}} \sim 20, I_{\mathrm{N}} \sim 19$ \\
\hline Pinfield et al. (1997) & INT/WFC & 1.0 & $\sim 10^{a}$ & $R=21.5, I=20.0, Z=21.5$ \\
\hline Magazzù et al. (1998) & INT/WFC & 0.22 & 1 & $R=22.2, I=21.2$ \\
\hline Chappelle et al. (2005) & INT/WFC & 2.6 & 4 & $I_{\mathrm{c}}=21 ., Z=20.5$ \\
\hline González-García et al. (2006) & $3.5 \mathrm{~m}$ CAHA / LAICA \& $5 \mathrm{~m} \mathrm{Hale} \mathrm{/} \mathrm{LFI}$ & 0.33 & 1 & $i^{\prime}=23.8, z^{\prime}=23.3$ \\
\hline Boudreault et al. (2010) & $3.5 \mathrm{~m}$ CAHA / $\Omega 2 \mathrm{k} \& 2.2 \mathrm{~m}$ La Silla/WFI & 3.1 & 6 & $I_{\mathrm{c}}=23.2, J=19.9, K s=18.6$ \\
\hline This work $^{b}$ & $\mathrm{LBT} / \mathrm{LBC}$ & 0.59 & 37 & $r=24.1, i=25.6, z=24.7, Y=20.3$ \\
\hline
\end{tabular}

Notes. The completeness limits correspond to a $10 \sigma$ detection, while those of the present work and Boudreault et al. (2010) correspond to $5 \sigma$ detections. ${ }^{(a)}$ From Fig. 3 in Pinfield et al. (1997), about 10 of their 26 Praesepe member candidates have masses below $72 M_{\text {Jup. }}{ }^{(b)}$ Our work was complemented by the the $J K_{\mathrm{s}} 3.5 \mathrm{~m}$ CAHA/ $\Omega 2 \mathrm{k}$ data from Boudreault et al. (2010).

unexplored mass regimes ( $\left.40 M_{\text {Jup }}\right)$. The main aims of our study are to search for new BDs and determine the MF of the Praesepe for a large coverage of the substellar regime.

The candidate selection procedure, and mass and temperature determination methods employed in this study are similar to those adopted in Boudreault et al. (2010). However, we probe a lower mass regime and use an evolutionary model based on a dusty atmosphere instead of a combination of dust-free and dusty models.

\section{Observations and analysis}

\subsection{Observations}

The observations presented in this paper were carried out with the Large Binocular Telescope (LBT) located on Mount Graham, Arizona (Hill et al. 2006), using the Large Binocular Cameras (LBCs, see Speziali et al. 2008). The LBCs are two wide-field, high-throughput imaging cameras, namely Blue (LBCB) and Red (LBCR), located at the prime focus stations of the LBT. Each LBC has a wide field of view $\left(23^{\prime} \times 23^{\prime}\right)$, with four CCD detectors of $2048 \times 4608$ pixels each, providing images with a sampling of $0.23^{\prime \prime} /$ pixel.

The optical design and detectors of the two cameras are optimized for different wavelength ranges: one for ultravioletblue wavelengths (320-500 nm, including the Bessel $U, B, V$ and Sloan $g$ and $r$ bands), and one for the red-infrared bands (500-1000 nm, including the Sloan $i, z$ and Fan $Y$ bands). In the full binocular configuration, both cameras are available simultaneously, and both point in the same direction of the sky, thus doubling the net efficiency of the LBT.

To accomplish the entire survey of the inner region of Praesepe, we carried out three observing runs, in March 2008, December 2008, and February 2009. Table 2 summarizes the observations and Fig. 1 shows the areas surveyed. The total area covered is $0.59 \mathrm{deg}^{2}$, about 1 percent of the cluster region. The transmission curves of the filters used in this survey is presented in Fig. 2, along with a synthetic spectrum of a brown dwarf with $T_{\text {eff }}=2300 \mathrm{~K}, \log g=4.5$ [CGS], and solar metallicity (NextGen model).

\subsection{Reduction and astrometry}

The standard data reduction steps for the LBT data were performed using the IDL astronomy package and IRAF. The bias subtraction was executed on a nightly basis and for each CCD chip. To correct for pixel-to-pixel variations and global illumination, master flat frames were created for the nights using twilight

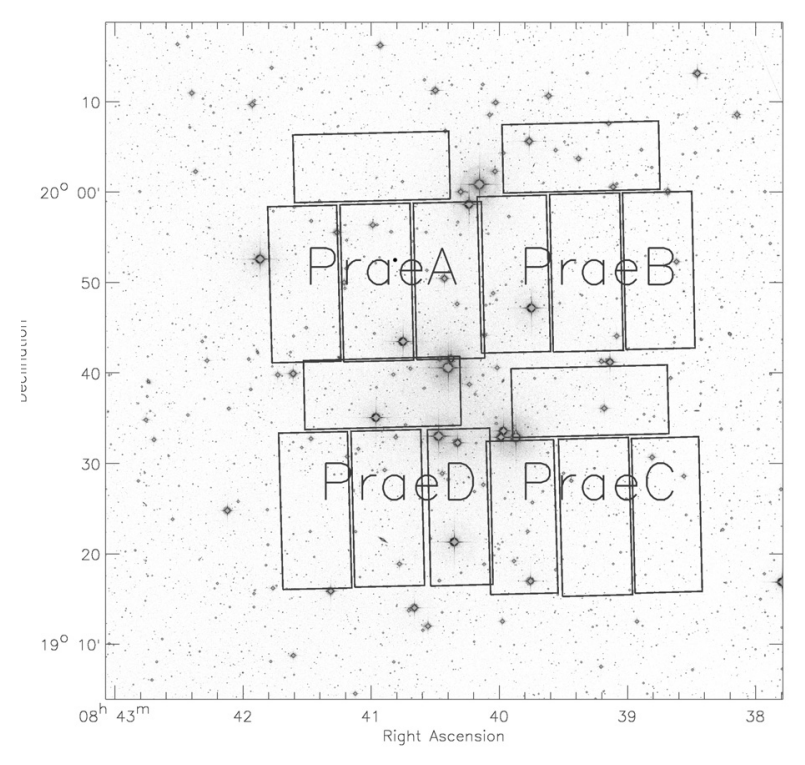

Fig. 1. Spatial distribution of the LBT pointings.

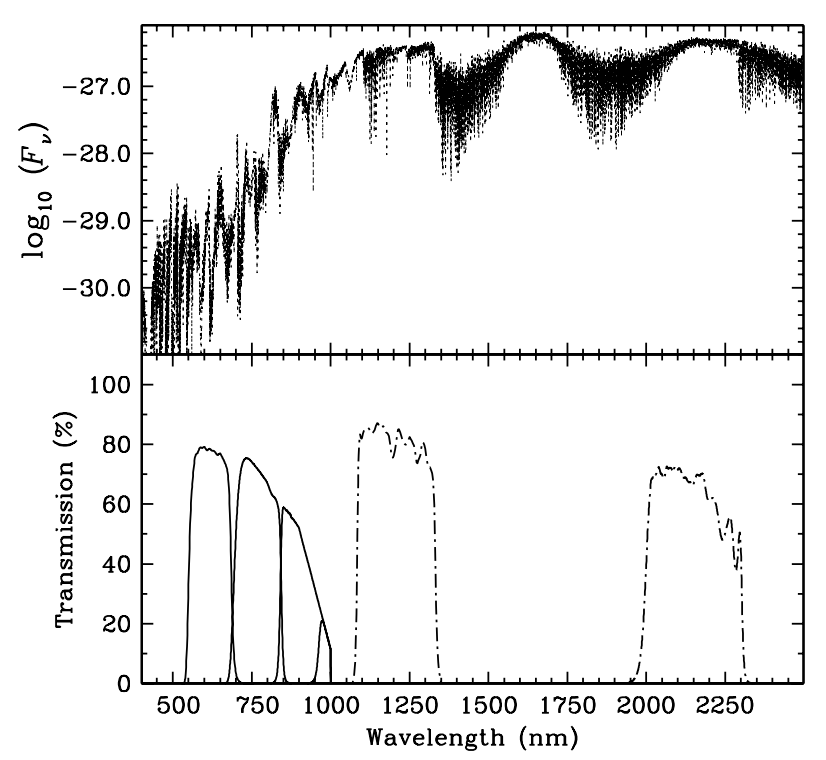

Fig. 2. Transmission curves of the filters used in our survey compared to a synthetic spectrum of a brown dwarf with $T_{\text {eff }}=2300 \mathrm{~K}, \log g=4.5$, and solar metallicity (NextGen model). The transmission curves include the quantum efficiency of the detectors. 
exposures. For nights when no appropriate sky flat exposures were available, we used a master sky flat in the adjacent night. The individual images of a given field were registered and median combined, resulting in a combined science frame for each CCD, field and filter. To detect faint sources, we subtracted the strong background introduced by very bright $\operatorname{stars}^{1}$. We then used the IRAF task daofind to detect sources in the "clean" frames. The sources were extracted from the original science frames and instrumental magnitudes calculated using both aperture and point-spread function photometry with the IRAF tasks phot and allstar respectively.

At this stage, weak fringes were still visible for several $Y$-band images. The method described by Bailer-Jones \& Mundt (2001) for removing fringes does not apply well in the present case, since the images are seriously affected by bright stars and no clean fringe images could be created. However, as the $Y$-band images are $\sim 1.5$ dex shallower than expected, we decided not to use them in this study (although we still quote some statistics of the photometry below).

An astrometric solution was achieved using the Sloan Digital Sky Survey (SDSS York et al. 2000) catalogue as a reference. The root mean square (rms) accuracy of our astrometric solution is $0.10-0.15$ arcsec. As with other reduction procedures, astrometry was also performed separately for each $\mathrm{CCD}$, to ensure that the solutions were as robust as possible.

\subsection{Photometric calibration}

To correct for Earth atmospheric absorption of the photometry, we calibrated the inferred data using the $r, i$, and $z$ band values of Sloan Digital Sky Survey (SDSS) objects that were observed in the science fields. Zero point offsets were determined from the difference between the SDSS magnitudes and our instrumental magnitudes. Since these were obtained with objects in the same field of view for each science frame, we did not perform a colour or airmass correction when reducing our riz photometry. The error introduced by this approximation is less than 0.05 mag.

To calibrate our $Y$ band photometry, we used our LBT $i$ and $z$ photometry and the $Y$ band photometry from the United Kingdom Infrared Telescope Infrared Deep Sky Survey (UKIDSS, Lawrence et al. 2007). We found that the differences between $Y$ band LBT magnitudes and $Y$ band UKIDSS magnitudes have a linear dependence on the $i-z$ colours, which can be described by the equation

$Y_{\mathrm{UKIDSS}}-Y_{\mathrm{LBT}, \mathrm{raw}}=a_{0}+a_{1} \times(i-z)$,

On the basis of about 800 common objects between UKIDSS and our measurements, the $\mathrm{a}_{0,1}$ coefficients were determined and the instrumental $Y$ magnitudes were then transferred into the UKIDSS $Y$ photometry system. For the same reasons as for our riz data, we did not (need to) perform a colour or airmass correction for our $Y$-band photometry.

The $5 \sigma$ detection limits of our survey are $\sim 25.6 \mathrm{mag}$ and $24.7 \mathrm{mag}$ for the $i$ and $z$ bands, respectively. However, we do not expect all targets brighter than these limits to be able to be detected. We estimate the survey completeness by comparing the number of objects detected to the number predicted assuming a uniform three-dimensional spatial distribution of stars. As shown in Fig. 3, the number of detected sources in each band deviates from a log-normal relationship at bright and faint limits. From this, we estimate the completeness to our $5 \sigma$ detection

\footnotetext{
1 The area fraction affected by bright stars is less than $3 \%$ for most of CCD images, and is about $6 \%$ in the worst case.
}

limit as $67.0 \%$ at $i=25.6$ and $70.3 \%$ at $z=24.7$ respectively, which corresponds to $\sim 40 M_{\text {Jup }}$ assuming a dusty atmosphere. A similar estimation yields a completeness of $82.0 \%$ at $r=24.1 \mathrm{mag}$ and $82.6 \%$ at $Y=20.3 \mathrm{mag}$, respectively. Our survey is approximately $95 \%$ complete at $i=23.8 \mathrm{mag}$ and $z=22.0$ mag.

The relatively low completeness of our survey is possibly caused by the saturation of bright stars. For stars lying near to saturated stars, the photometric uncertainties are relatively large. A significant fraction of detected stars is then excluded because of their large photometric errors, which lowers the completeness. The total area seriously affected by bright stars in $i$ band is $3-6$ percent.

\subsection{Candidate selection procedure}

The candidate selection introduced by Boudreault et al. (2010) was adopted in the present work. Candidates were first selected based on the colour-magnitude diagram (CMD) using iz bands from our LBC observations ${ }^{2}$ A second selection was performed using a colour-colour diagram. While our $r Y$ bands observations are not deep enough for our present investigations, data from the near-IR photometric survey by Boudreault et al. (2010) which fully covers our survey area, with a $5 \sigma$ detection limit at $\sim 55 M_{\text {Jup }}$ in $J$ and $K_{\mathrm{s}}$ bands - was used instead for the second selection. In the third and final selection, we used the known distance to Praesepe to reject objects based on the discrepancy between their observed magnitude in $J$ and the magnitude predicted from the isochrones and our estimation of $T_{\text {eff }}$. To be considered as a cluster member, an object had to satisfy all three of these criteria.

We use the evolutionary tracks of Chabrier et al. (2000) and the atmosphere models from Allard et al. (2001) - assuming a dusty atmosphere (the AMES-Dusty model) - to compute an isochrone for Praesepe using an age of $590_{-120}^{+150}$ Myr (Fossati et al. 2008), a distance of $190_{-5.8}^{+6.0} \mathrm{pc}$ (van Leeuwen 2009), and a solar metallicity $([\mathrm{Fe} / \mathrm{H}]=0.038 \pm 0.039$, Friel \& Boesgaard $1992)$. We neglect the reddening $(E(B-V)=0.027 \pm 0.004 \mathrm{mag}$, Taylor 2006). The transmission curves we used for the filters for these calculations are plotted in Fig. 2. The effective temperature varies from $500 \mathrm{~K}$ to $3900 \mathrm{~K}$ in steps of $100 \mathrm{~K}$, while the gravity ranges from 4.0 dex to 6.0 dex in steps of 0.5 dex.

\subsubsection{Colour-magnitude diagram}

Candidates were first selected from our CMD by keeping all objects that are no more than 0.28 mag redder or bluer than the isochrones in all CMDs. This number accommodates errors in the magnitudes and uncertainties in the model isochrones. We also include the errors from the age estimate and distance to Praesepe. We additionally include objects brighter than the isochrones by $0.753 \mathrm{mag}$ in order to include unresolved binaries. In Fig. 4, we show the CMD where candidates were selected based on their values of $z$ versus $i-z$.

From a total of 44209 objects above the $5 \sigma$ detection limit in $i$ and $z$ filters and below our saturation limit, 709 objects are retained as candidate cluster members ( $98.4 \%$ are rejected). Of these, 160 were detected in the NIR observations of Boudreault et al. (2010). These objects are used in the selection process described below.

\footnotetext{
2 Our $r Y$ bands observations do not reach a similar stellar mass, hence are not used here.
} 

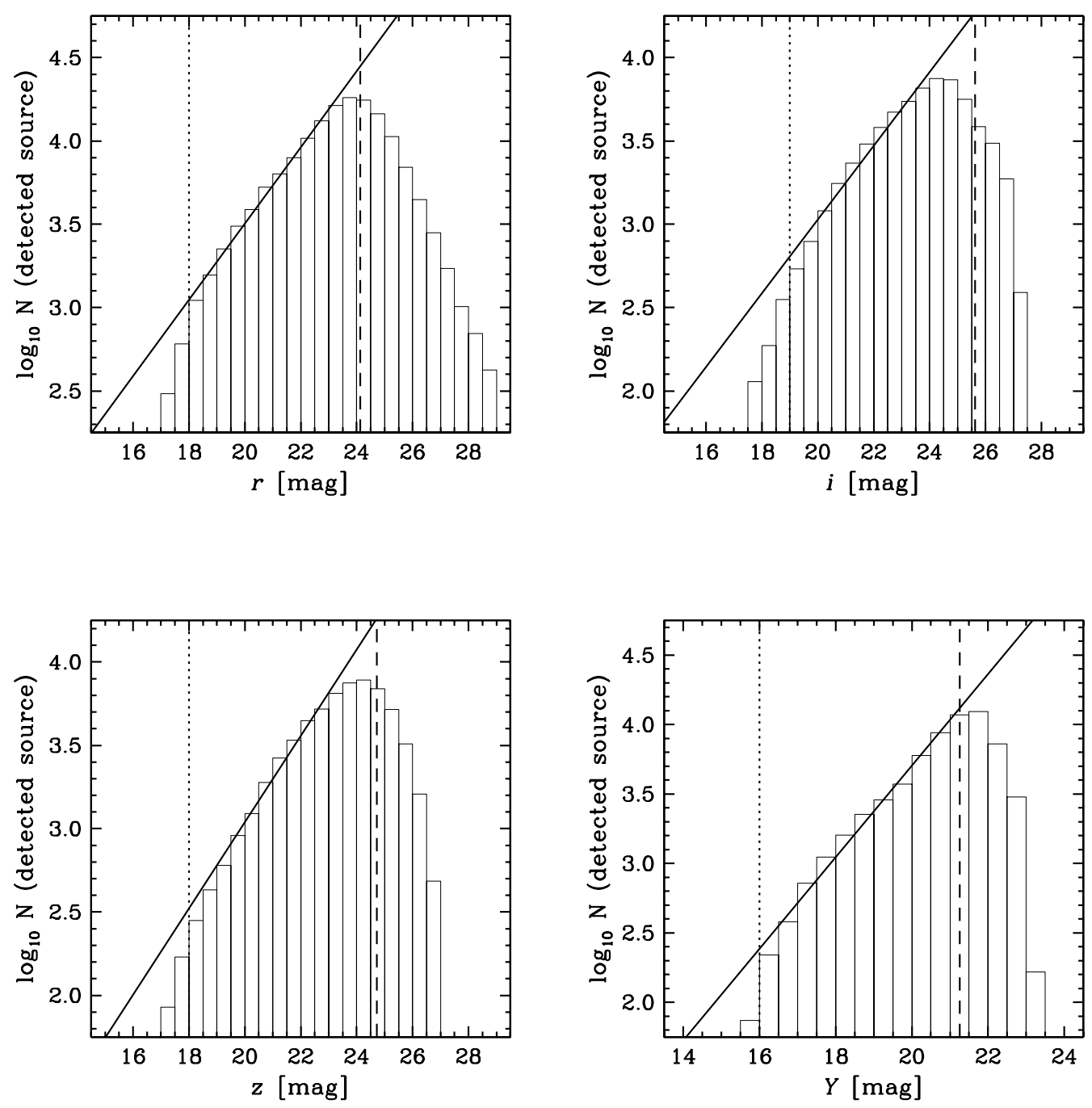

Fig. 3. Estimation of the completeness limit for our survey using the $i z$ bands. The solid lines are the best linear fits before the turn offs. The vertical dashed lines are the $5 \sigma$ detection limit and the vertical dotted lines are the saturation magnitudes.

\subsubsection{Colour-colour diagram}

From this step, The candidate selection is based on both the optical $i z$ data and the NIR $J K_{\mathrm{S}}$ data. A candidate must be detected in every band.

The second stage of the candidate selection involves retaining only those objects that lie within $0.28 \mathrm{mag}$ of the isochrone in the colour-colour diagram. This value accommodates the photometric errors, uncertainties in the model isochrones, and the uncertainty in the age estimation of Praesepe. The colour-colour diagram with the selection limits is shown in Fig. 5, where we also plot the theoretical colours of red giants (using the atmosphere models of Hauschildt et al. 1999b) and the theoretical colours of six galaxies with redshifts from 0 to 2 (Meisenheimer et al., in prep.). Neither the red giants nor the galaxies are expected to be a significant source of contamination; most of the low redshift galaxies that were not automatically discarded during PSF photometry with full-width-half-maxima $(F W H M$ s $)$ broader than the average stellar $F W H M$ by $\sim 30 \%$, were rejected by means of the visual inspection of individual cluster member candidates after selection procedures. Of the 160 objects selected in the first step, 88 are kept here.

\subsubsection{Observed magnitude vs. predicted magnitude}

As indicated in Sect. 3.2, our determinations of $T_{\text {eff }}$ and mass are based on the spectral energy distribution of each object, so are independent of the assumed distance. The membership status of an object can therefore be assessed by comparing its observed magnitude in a band with its magnitude predicted from its $T_{\text {eff }}$ and Praesepe's isochrone (which assumes a distance). The predicted magnitude of a background contaminant would be lower (brighter) than its observed magnitude and higher (fainter) for a foreground contaminant. To avoid removing unresolved binaries that are real members of the cluster, we keep all objects with a computed magnitude of up to 0.753 mag brighter than the observed magnitude. We also take into account photometric errors and uncertainties in the age and distance of Praesepe. This selection procedure is illustrated in Fig. 6.

Of the 88 objects selected through CMDs and colour-colour diagrams in the first two steps, 74 are retained here. After this step, we perform a direct visual inspection of the images to reject resolved galaxies and spurious detections. This inspection removes 15 objects from the photometric selection, of which seven are possibly galaxies and eight are false detections.

The remaining objects constitute our final cluster member candidates, shown as large dots in Fig. 7. Those selected using 
W. Wang et al.: Mass function of Praesepe from deep LBT observations

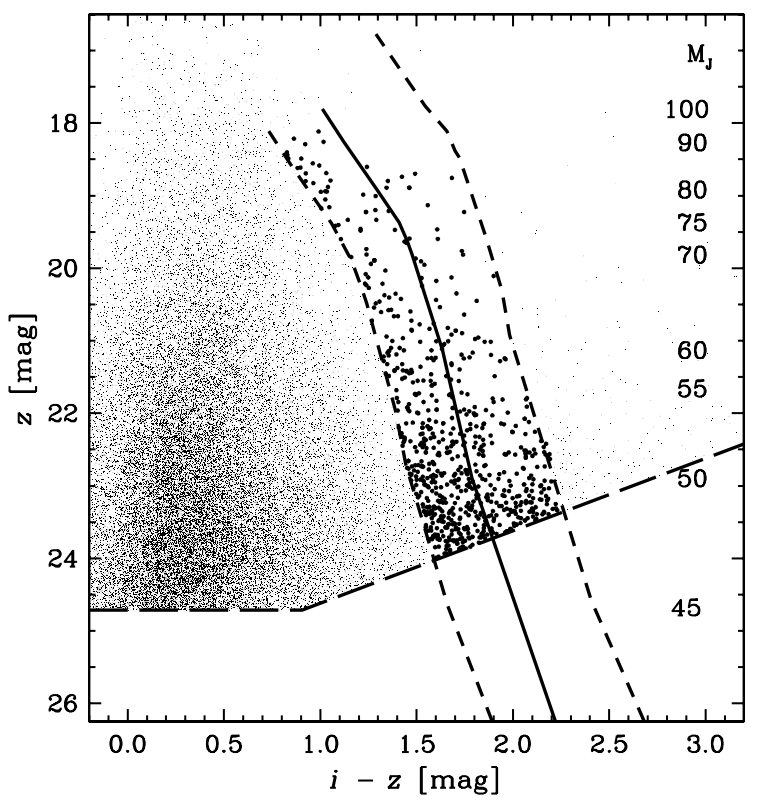

Fig. 4. CMD with $i$ and $z$ bands used in the first selection procedure. As solid lines we show the isochrone computed from an evolutionary model with a dusty atmosphere (AMES-Dusty). The numbers indicate the masses (in $M_{\text {Jup }}$ ) on the model sequence for various $z$ magnitudes. The dashed lines delimit our selection band.

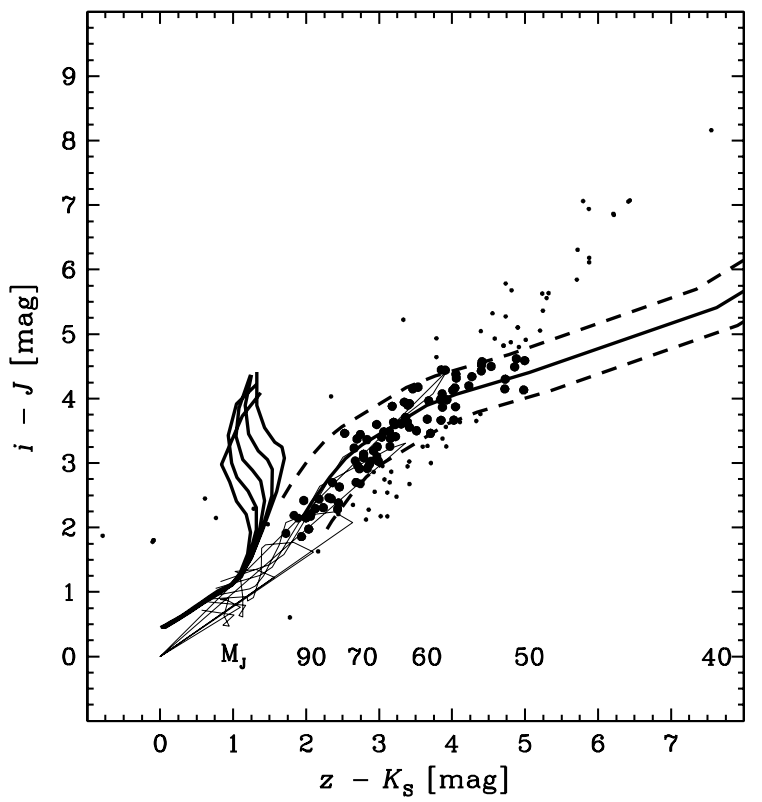

Fig. 5. Colour-colour diagram used in the second selection step. The solid line is the isochrone computed from an evolutionary model with a dusty atmosphere (the AMES-Dusty model; the masses in $M_{\odot}$ for each $z-K_{\mathrm{s}}$ colour are shifted for clarity). The dashed lines show our selection area. We also show the theoretical colours of six galaxies as thin lines and the theoretical colours of red giants as thick lines. The six galaxies are two starbursts, one Sab, one Sbc, and two ellipticals of 5.5 and $15 \mathrm{Gyr}$, with redshifts from $z=0$ to $z=2$ in steps of 0.25 (evolution not considered). We assume that all red giants have a mass of $5 M_{\odot}, 0.5<\log g<2.5$ and $2000 \mathrm{~K}<T_{\text {eff }}<6000 \mathrm{~K}$.

only the iz photometry amount to 709, and are presented in Fig. 7 as small dots. We note that employing NIR $J K_{\mathrm{s}}$ data helps us to remove a significant fraction of contaminations. At each mass bin, we calculate the number of stars removed as a result of including $J K_{\mathrm{S}}$ data in the selection, and use this to estimate the

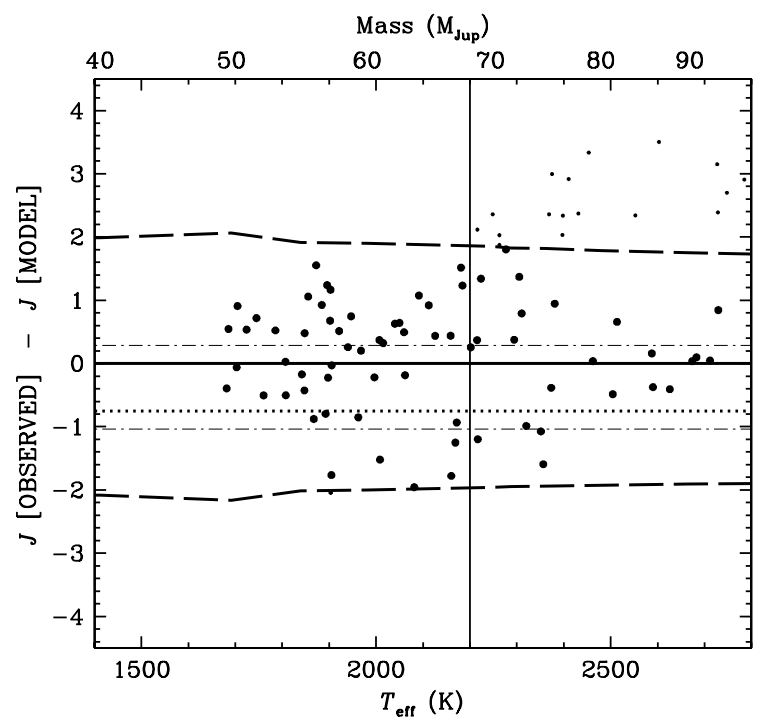

Fig. 6. Difference between the observed $J$ magnitude and the model $J$ magnitude computed from the derived mass and $T_{\text {eff }}$, as a function of $T_{\text {eff }}$. The vertical line marks the location of L0 dwarfs. The dotted line (at $-0.753 \mathrm{mag}$ ) represents the offset due to the possible presence of unresolved binaries, the dashed-dotted lines represent the error in the magnitude determination, and the long-dashed lines represent the uncertainties in the age and distance of Praesepe. The horizontal solid line just traces zero.

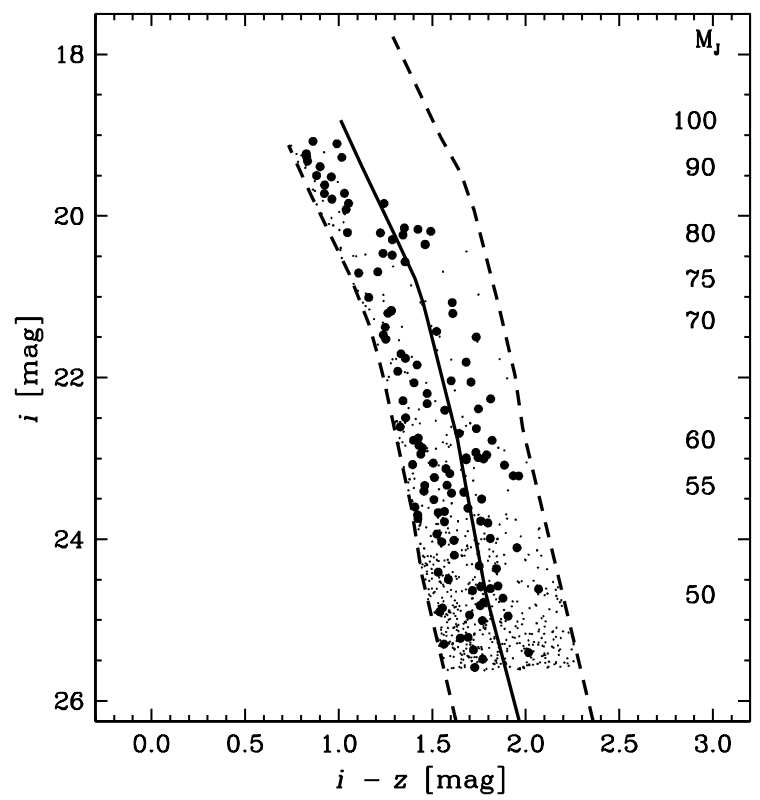

Fig. 7. Same as Fig. 4, but with large dots standing for the 59 final candidates, and small dots for the 709 candidates that pass the first selection procedures.

number of stars in the final mass bin where $J K_{\mathrm{s}}$ data are unavailable.

Schmidt et al. (2010) and West et al. (2011) investigated the colors of L and M dwarfs, respectively, for every spectral types using the SDSS and Two Micron All Sky Survey (2MASS) catalogues. The observed colour ranges are consistent with our computed colour ranges for $\mathrm{M}$ and early $\mathrm{L}$ dwarfs. However, the observed $i-J$ color range for individual $\mathrm{L}$ dwarfs as shown in Fig. 2 in Schmidt et al. (2010) is 3.5-6.0 mag, broader and slightly redder than our model ranges (the dashed lines in our Fig. 5). We note that the Hammer spectral-typing procedure employed by 
Schmidt et al. (2010), was developed by Covey et al. (2007), who sought to optimize only for K and M dwarfs. For L dwarfs, the uncertainties should be $\sim 2$ subclasses or larger. In addition, the Hammer is designed for solar-metallicity dwarfs, while Praesepe has a metallicity of $0.27 \pm 0.10$ dex (Pace et al. 2008), hence will be prone to significant, systematic errors (Covey et al. 2007). We therefore keep the use of our model colours alone for candidate selection for a homogeneous study. We found that if we use the observed color range from Schmidt et al. (2010) instead, the observed rise in the mass function around $60 M_{\text {Jup }}$ (cf. Sect. 4) remains - our main conclusion remains unchanged.

\section{Results}

\subsection{Selected photometric candidates}

We find that 59 photometric candidates survive the selection procedures (based on isochrones assuming dusty atmospheres), a density of about 100 objects per square degree. Details of all photometric candidates are listed in Table 3. The identification number (ID) of a candidate is defined according to the field in which it was found and a sequential number for that field. The last column, $J_{\text {model }}$, is the predicted $J$ magnitude based on photometric determination of $T_{\text {eff }}$ and mass.

Our survey concentrates on the substellar regime. Saturation occurs at $\sim 18 \mathrm{mag}$ in $z$ band, corresponding to $\sim 100 \mathrm{M}_{\text {Jup }}$. Therefore, most of the low mass candidates discovered in previous surveys (e.g. Pinfield et al. 1997; Hambly et al. 1995) are saturated in our LBT images. Only a few faint brown dwarfs classified by Pinfield et al. (1997), González-García et al. (2006), and Boudreault et al. (2010) are rediscovered in the current survey (cf. Table 4). These objects are M 5-9 dwarfs, according to the photometric relations given by West et al. (2008).

Some of our targets were previously identified as cluster members but rejected by our selection procedures or visual inspection. For example, eleven of the 150 Boudreault et al. (2010) candidates are detected in our LBT survey (the rest are mostly saturated). However, nine of them are rejected in this work (cf. Table 5). Among them, seven are rejected on the basis of the $z$ vs. $i-z$ CMD selection, because they are bluer than the isochrone area. Another one is obviously not a point-like source in the LBT image, and another is rejected because its observed $J$ magnitude is inconsistent with its model-predicted magnitude. The remaining two targets are confirmed to be cluster dwarf stars. As our current work employed more photometric bands than Boudreault et al. (2010), it is unsurprising that our selection is more conservative.

Most of our candidates are in the substellar regime, and other than the five targets listed in Table 4, no other accurate photometric observations are available from past epochs. This precludes using proper motions as a means of cluster membership assessment at this time.

\subsection{Photometrically-derived masses and effective temperatures}

From the evolutionary tracks and atmosphere models described previously, we obtained the magnitudes $m_{\mathrm{A}}$ from the average flux of a star in a specific band $A$ using the equation

$m_{\mathrm{A}}=-2.5 \log f_{\mathrm{A}}+c_{\mathrm{A}}$,

where $m_{\mathrm{A}}$ is the magnitude observed in a given passband, $f_{\mathrm{A}}$ the flux received on Earth in this passband, and $c_{\mathrm{A}}$ is a constant that remains to be determined. The flux $f_{\mathrm{A}}$ was obtained
Table 4. Previously discovered low-mass Praesepe candidates found in our survey.

\begin{tabular}{lllll}
\hline \hline ID & $\begin{array}{l}\text { RA(J2000) } \\
(\mathrm{h} \mathrm{m} \mathrm{s})\end{array}$ & $\begin{array}{l}\text { Dec(J2000) } \\
\left({ }^{\circ}{ }^{\prime \prime \prime}\right)\end{array}$ & Alternative name & Ref. $^{a}$ \\
\hline A16 & $8: 41: 24.916$ & $19: 57: 26.19$ & NGC2632 PHJ20 & $(1)$ \\
A17 & $8: 41: 26.483$ & $19: 51: 59.73$ & J084126.5+195200 & $(2)$ \\
C12 & $8: 39: 47.778$ & $19: 28: 03.10$ & Praesepe017 & $(3)$ \\
& & & NGC2632 PHJ11 & $(1)$ \\
D12 & $8: 40: 53.559$ & $19: 41: 00.05$ & Praesepe001 & $(3)$ \\
D08 & $8: 40: 39.292$ & $19: 28: 39.49$ & J084039.3+192840 & $(2)$ \\
\hline
\end{tabular}

References. (1) Pinfield et al. (1997); (2) González-García et al. 2006; (3) Boudreault et al. (2010).

using the total transmission function of the passband for a given filter, convolved with the quantum efficiency of the CCDs (we assumed that the telescope and instrumental throughput is flat over each passband). To transform our optical $i$ and $z$ band magnitudes from the models to the Johnson photometric system, we assumed all magnitudes $m_{\mathrm{A}}$ to be equal to 0.03 when $f_{\mathrm{A}}$ is the average Vega flux received on Earth. The constant $c_{\mathrm{A}}$ for each passband is then determined using the Vega flux from Colina et al. (1992). The fitted values for these constants are $i=-22.180$ and $z=-22.706 \mathrm{mag}$. The values computed by Boudreault et al. (2010) for $J$ and $K_{\mathrm{s}}$ were -23.687 and $-25.908 \mathrm{mag}$, respectively.

The masses and effective temperatures were estimated in the way described by Boudreault \& Bailer-Jones $(2009)^{3}$. For the faintest objects where only $i$ and $z$ are available, the colour $i-z$ is used to compute masses and $T_{\text {eff }}$. There are different sources of errors for the estimation of the mass and $T_{\text {eff }}$, including the photon noise, the photometric calibration, the least squares fitting (imperfect model), and the uncertainties in the age of and distance to Praesepe. The latter two are the most significant errors and give the uncertainties of $0.008 M_{\odot}$ and $263 \mathrm{~K}$ for a $0.05 M_{\odot}$ substellar object $\left(T_{\text {eff }}=1690 \mathrm{~K}\right), 0.010 M_{\odot}$ and $260 \mathrm{~K}$ for a $0.06 M_{\odot}$ substellar object $\left(T_{\text {eff }}=1990 \mathrm{~K}\right)$, and $0.008 M_{\odot}$ and $201 \mathrm{~K}$ for a $0.072 M_{\odot}$ object at the hydrogen burning limit $\left(T_{\text {eff }}=2293 \mathrm{~K}\right)$.

\subsection{Contamination by non-members}

As mentioned above, the three main sources of contamination are background red giants, unresolved galaxies, and Galactic $\mathrm{M}$ and L dwarfs. Red giants contaminate the high mass end of this study, as seen in the $i-J$ vs. $z-K_{\mathrm{s}}$ diagram, hence can be ignored. Although some types of galaxies have similar colours to Praesepe cluster members more massive than $60 M_{\text {Jup }}$, these low-redshift galaxies are in general extended sources and therefore easily rejected by our visual inspection. Among the 74 candidates that passed our selection procedures, we identify four as galaxies on the basis of their LBT images. Other possible sources are field L dwarfs and high redshift quasars (for instance at redshift $z \sim 6$; Caballero et al. 2008). However, because these quasars have spectral energy distributions similar to mid-T dwarfs, whereas our faintest candidates have colours of early L dwarfs, and given that they are rare ( 0.25 quasars at

\footnotetext{
3 We first normalized the energy distribution of each object to the energy distribution of the model using the $J$ filter. The energy distribution was then fitted via a least squares fit of the model magnitudes to the measured ones.
} 
W. Wang et al.: Mass function of Praesepe from deep LBT observations

Table 5. The 9 Praesepe cluster candidates identified by Boudreault et al. (2010) that were rejected in this work by colour or brightness.

\begin{tabular}{ccccccccccc}
\hline \hline ID & RA(J2000) & $\operatorname{Dec}(\mathrm{J} 2000)$ & $\begin{array}{c}i \\
(\mathrm{mag})\end{array}$ & $\begin{array}{c}z \\
(\mathrm{mag})\end{array}$ & $\begin{array}{c}I_{\mathrm{c}} \\
(\mathrm{mag})\end{array}$ & $\begin{array}{c}J \\
(\mathrm{mag})\end{array}$ & $\begin{array}{c}K_{\mathrm{s}} \\
(\mathrm{mag})\end{array}$ & $\begin{array}{c}M \\
\left(M_{\odot}\right)\end{array}$ & $\begin{array}{c}T_{\text {eff }} \\
(\mathrm{K})\end{array}$ & $\begin{array}{c}J_{\text {model }} \\
(\mathrm{mag})\end{array}$ \\
\hline 005 & $8: 41: 08.50$ & $+19: 54: 02.0$ & 20.19 & 18.70 & 19.02 & 16.58 & 15.39 & 0.088 & 2636 & 17.06 \\
007 & $8: 39: 39.56$ & $+19: 47: 54.3$ & 18.93 & 17.80 & 17.95 & 16.10 & 15.07 & 0.104 & 2860 & 16.58 \\
009 & $8: 39: 55.84$ & $+19: 53: 14.3$ & 18.05 & 17.78 & 20.29 & 17.50 & 16.54 & 0.081 & 2520 & 17.32 \\
018 & $8: 39: 42.79$ & $+19: 35: 48.2$ & - & - & 18.27 & 16.20 & 15.21 & 0.097 & 2782 & 16.78 \\
022 & $8: 41: 04.20$ & $+19: 31: 27.8$ & 20.12 & 19.15 & 18.89 & 16.67 & 15.75 & 0.092 & 2702 & 16.97 \\
901 & $8: 39: 59.45$ & $+19: 43: 37.4$ & 18.05 & 18.30 & 19.09 & 17.16 & 16.41 & 0.084 & 2574 & 17.20 \\
902 & $8: 39: 23.72$ & $+19: 52: 01.8$ & 20.41 & 19.46 & 20.15 & 17.77 & 16.88 & 0.073 & 2348 & 17.72 \\
903 & $8: 40: 00.20$ & $+19: 30: 27.0$ & 19.59 & 19.01 & 19.74 & 17.50 & 16.62 & 0.076 & 2409 & 17.57 \\
914 & $8: 38: 52.02$ & $+19: 35: 05.3$ & 19.48 & 18.69 & 19.12 & 17.25 & 16.35 & 0.085 & 2591 & 17.16 \\
\hline
\end{tabular}

Notes. The IDs in the first column are taken from Boudreault et al. (2010). The fourth object is not a point-like source in the LBT image, thus no optical photometry measurement is available.

Table 6. Number density of the cluster member candidates.

\begin{tabular}{cccccc}
\hline \hline $\begin{array}{l}\log M \\
\left(M_{\text {Jup }}\right)\end{array}$ & $\begin{array}{c}T_{\text {eff }} \\
(\mathrm{K})\end{array}$ & $\begin{array}{c}J_{\text {model }} \\
(\mathrm{mag})\end{array}$ & $\begin{array}{c}N(\text { cand. }) \\
\left(\mathrm{deg}^{-2}\right)\end{array}$ & $\begin{array}{c}N(\text { cont. }) \\
\left(\mathrm{deg}^{-2}\right)\end{array}$ & Fraction \\
\hline 1.625 & 1412 & 23.29 & 13 & 195 & $1500 \%$ \\
1.675 & 1692 & 20.28 & 27 & 41 & $150 \%$ \\
1.725 & 1839 & 19.27 & 18 & 3.3 & $15 \%$ \\
1.775 & 1981 & 18.87 & 33 & 4.8 & $13 \%$ \\
1.825 & 2244 & 18.01 & 38 & 2.3 & $5.7 \%$ \\
1.875 & 2361 & 17.71 & 17 & 3.2 & $16 \%$ \\
1.925 & 2479 & 17.43 & 8.4 & 1.7 & $17 \%$ \\
1.975 & 2668 & 17.01 & 8.4 & 1.6 & $16 \%$ \\
\hline
\end{tabular}

$5.5<z<6.5$ in a $0.59 \mathrm{deg}^{2}$ survey, Stern et al. 2007), the MF should not be affected by quasar contamination.

The contamination by field dwarfs is not negligible. Caballero et al. (2008) identified possible field dwarf contaminants covering spectral types from M 3 to T8 from the literature and presented the spatial density in the solar neighbourhood in their Table 3. From this, the spatial density of field dwarfs in the vicinity of Praesepe can be easily inferred, given the Galactic latitude of Praesepe of $b=+32.5 \mathrm{deg}$ and its distance of $190 \mathrm{pc}$, assuming an exponential decrease for stellar density perpendicular to the Galactic disk with a scale height of $500 \mathrm{pc}$. The absolute $J$ band magnitude range constrained by our selection procedures is $\sim \pm 2$ mag, as shown in Fig. 6, corresponding to a certain distance interval and a survey "volume", which is defined by the product of survey area and depth. We calculated the number of contaminants by multiplying the survey volume by spatial density at each mass bin. The result is shown in Table 6. The first column gives the central value of $\log M$ in each interval, while the second and third columns present the corresponding $T_{\text {eff }}$ and $J_{\text {model }}$ values at that mass. The fourth column is the number density of cluster member candidates (also show in Fig. 8 as filled triangles) after applying all corrections (except for in the lowest two bins, where contaminations are too high to be corrected). The final two columns give the number density of field dwarf contaminants and corresponding fraction. We found that the field dwarf contaminants do not affect the MF shape. The contamination is significant for $J_{\text {model }} \gtrsim 20 \mathrm{mag}$. At this magnitude, the Boudreault et al. (2010) $J$ band has a completeness of $88 \%$. Therefore, below this magnitude, the mass functions we derived are probably upper limits.

We conclude that the various contaminants are either negligible, or do not affect the MF shape in the range that we can investigate quantitatively, i.e., from about 53 to $94 M_{\text {Jup }}$.

\section{Mass function of very low mass and substellar population of Praesepe}

The mass function, $\xi\left(\log _{10} M\right)$, we present here is the total number of objects per square degree in each logarithmic mass interval $\log _{10} M$ to $\log _{10} M+0.1$. Since we do not make any corrections for binaries, we compute here a system MF.

As our candidates have been selected only from their photometric properties, cluster membership confirmation via spectroscopy is desirable. However, these observations will not be feasible in the near future because of the faintness of our candidates. The following discussion is therefore based on the assumption that the MF of candidates is similar to that of "real" cluster members. The assumption is possibly valid because our derived MF is consistent with that given by Boudreault et al. (2010) in the common mass bin, and the contamination by field dwarfs, giants, and galaxies should not affect the shape of the MF significantly.

To account for the survey detection efficiency, we use a simple simulation. For example, to calculate the detection efficiency of candidate A01 in $i$ band, we select a bright but unsaturated star in the $i$ band CCD image in which A01 resides, scale it down to the magnitude of A01 (i.e., $i=20.29$ ), and randomly cast this "fake" star in the CCD image 100 times. We then search and re-measure the "fake" star with our procedures. The detection efficiency is the fraction of "fake" stars that have been rediscovered. We run this test for each filter $\left(i z J K_{\mathrm{s}}\right)$ and multiply the detection efficiencies together (as we need a detection in every filter) to evaluate the overall detection efficiency for each candidate. This detection efficiency (or recovery rate) is about $90 \%$ for the brightest candidates and drops very quickly to $10 \%$ for the faintest candidates.

We mentioned in Sect. 2 that our optical photometry reaches lower masses than the NIR photometry that we used. To compute the MF of Praesepe to the lowest mass bin reached without optical data, we first computed a $\mathrm{MF}^{4}$ using only the optical $i z$ photometry. This "MF' is presented in Fig. 8 as filled dots. We computed a second MF from the list of candidates that pass the three selection criteria and are also detected in the survey of Boudreault et al. (2010) in the NIR $J$ and $K_{\mathrm{s}}$ bands (presented on Fig. 8 as filled triangles). For each mass bin, we computed the number of objects removed as a result of adding the $J$ and $K_{\mathrm{S}}$ filters to our selection process and mass determination procedure (plotted as a function of mass in Fig. 8, top panel). We fitted a linear function to estimate the number of objects that

${ }^{4}$ Note that this is an inaccurate "MF", because of serious contaminations. 


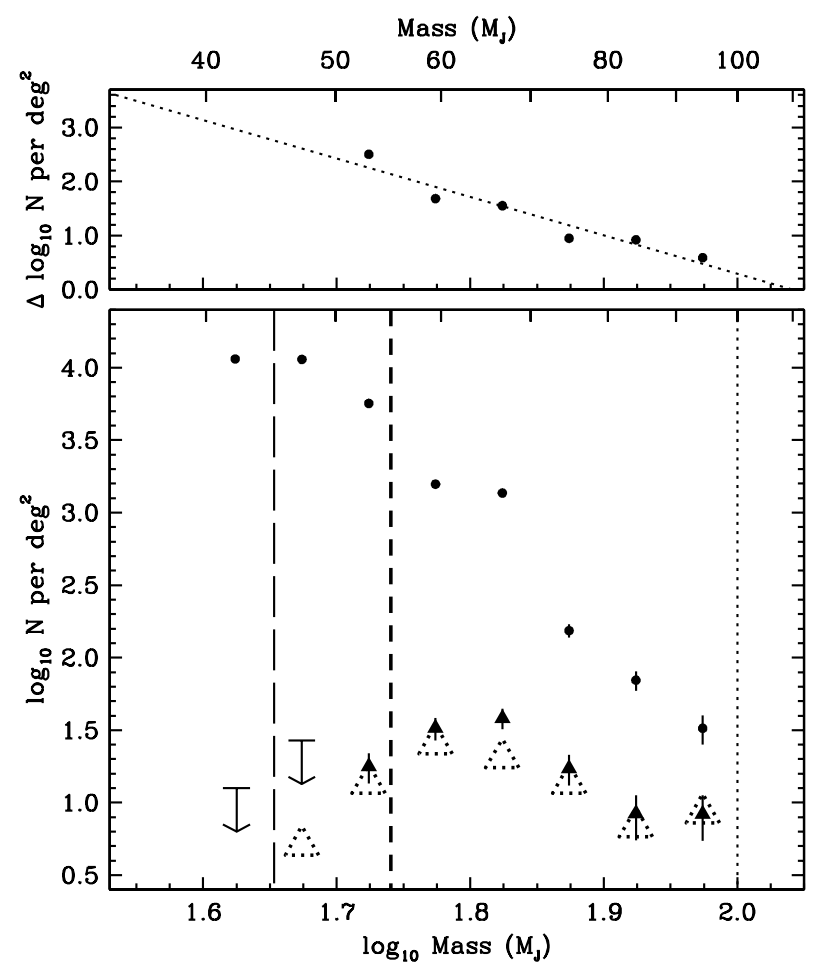

Fig. 8. Lower panel. Mass function based on a) the LBT $i$ and $z$ photometry (filled dots) after detection efficiency corrections, and b) the LBT photometry plus the $J$ and $K_{\mathrm{s}}$ photometry from Boudreault et al. (2010) (dotted open triangles: the original MF; filled triangles: the MF after detection efficiency corrections and removing contaminants). The selection and mass calibration are based on dusty atmospheres. Error bars are Poissonian arising from the number of objects in each bin, except for the lowest-mass bin, for which the error bar is from the linear fit in the top panel. The vertical thin dotted lines are the mass limits at which detector saturation occurs in the $i$ and $z$-bands. The vertical thin long dashed line is the mass at the $5 \sigma$ detection limit of our optical LBT data while the thick short dashed line is the mass at the $5 \sigma$ detection limit of the NIR data of Boudreault et al. (2010). Top panel. Difference of the $\log$ of the number of objects (in each mass bin) between the MF computed using the optical $i z$ data and the MF computed using both $i z$ and NIR $J K_{\mathrm{s}}$ data from Boudreault et al. (2010). The dotted line is a linear fit.

would be removed if we had additional $J$ and $K_{\mathrm{s}}$ photometry to 40-45 $M_{\text {Jup }}$, which is our lowest mass data point in Fig. 8. However, as shown in Table 6, the contamination in the two lowest mass bins is so overwhelming that the MFs in these two bins can only be regarded as upper limits and are no longer discussed in the paper.

Our derived MF (presented in Figs. 8-10) shows a rise from $105 M_{\text {Jup }}$ to $67 M_{\text {Jup }}$ and then a turn-over at $\sim 67 M_{\mathrm{J}}$. This turnover occurs well above the $5 \sigma$ of either $i z$ bands or $J K_{\mathrm{s}}$ bands (e.g. at $\sim 67 M_{\text {Jup }}, i \sim 22$ ) and we note that we have corrected the incompleteness of our survey and field dwarf contaminations. We therefore believe that this feature is genuine. This is the first time a clear rise in the substellar MF in an old open cluster has been observed.

The MF of Praesepe near the hydrogen-burning limit was previously obtained in several studies. However, only Boudreault et al. (2010) provide a common mass range for comparison, as shown in Fig. 9. In the first substellar mass bin $\left(\sim 80 M_{\text {Jup }}\right)$ we see that, both surveys give consistent results within their error bars. However, for the second bin at $\sim 70 \mathrm{M}_{\mathrm{Jup}}$, our MF is much higher than that of Boudreault et al. (2010); the

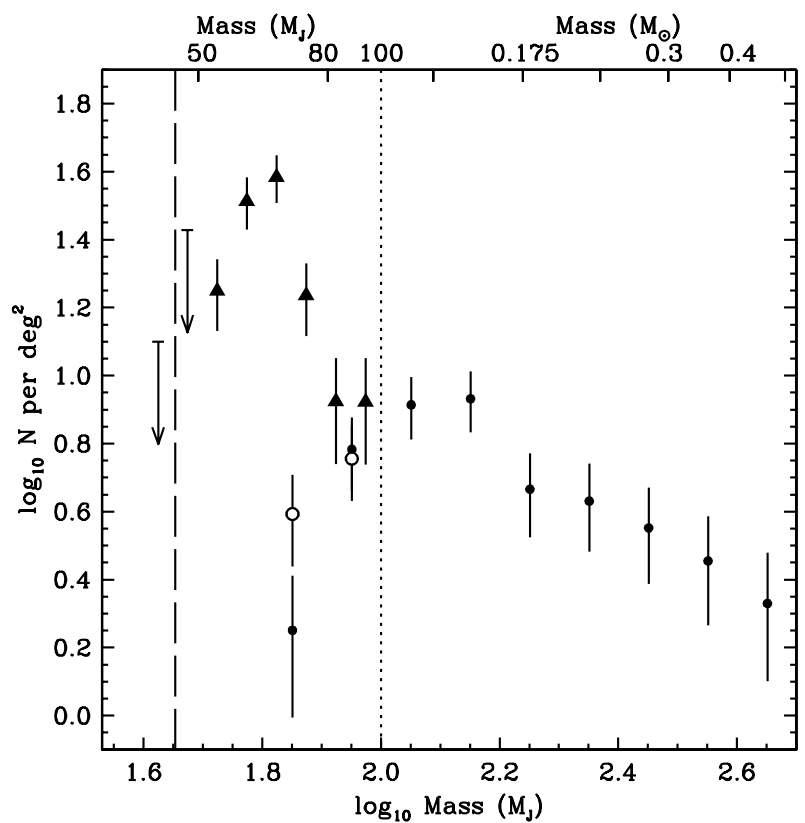

Fig. 9. MF of Praesepe based on our survey LBT $i z$ and $\Omega 2 \mathrm{k} J K_{\mathrm{s}}$ photometry (triangles), compared with that from Boudreault et al. (2010) (open dots assuming a dusty atmosphere and filled dots assuming a dustfree atmosphere). Error bars are Poissonian arising from the finite number of objects observed in each bin, except for the last bin, for which the error bar is derived from the linear fit. The vertical thin dotted line is the mass limit above which detector saturation occurs in the $i$ and $z$-bands. The vertical thin long-dashed line is the mass at the $5 \sigma$ detection limit of our optical LBT data.

discrepancy is smaller when considering the MF from that work using the dusty atmosphere (open dots), which is still a reasonable model for such low mass stars $(\sim \mathrm{M} 9 / \mathrm{L} 0)$. This may indicate that some faint candidates are missing in the Boudreault et al. (2010) survey, as these authors did not make any corrections for the detection efficiencies.

We emphasize that our LBT survey covers the very central $0.59 \mathrm{deg}^{2}$ of Praesepe, while the $\Omega 2 \mathrm{k}$ survey by Boudreault et al. (2010) covers a much wider area $\left(\sim 3.1 \mathrm{deg}^{2}\right)$. If no significant candidates are missing in the $\Omega 2 \mathrm{k}$ survey, this discrepancy may suggest that the very low mass cluster members are mostly concentrated in the cluster centre, in contrast to what is expected from a "dynamical evaporation" of brown dwarf in open clusters. The basic idea of dynamical evaporation is that lower mass stars in a cluster have higher speeds according to equipartition of energy, so are able to move higher in the gravitational potential well of the cluster. Hence the fraction of low mass stars should increase with increasing distance from the cluster centre. By comparing the Praesepe and Hyades MFs, Boudreault et al. (2010) concluded that Praesepe might have been less affected by dynamical evolution.

Owing to its large distance and old age, no other published MF determination of Praesepe has reached masses below $70 M_{\text {Jup }}$. We therefore compare our results with those from other clusters in Fig. 10. This includes IC 2391 from Boudreault \& Bailer-Jones (2009), ONC from Hillenbrand \& Carpenter (2000), $\sigma$ Orionis from (Caballero et al. 2007; Bihain et al. 2009), and the Hyades from Bouvier et al. (2008). The MF of Praesepe is quite different from both IC 2391 (age of 50 Myr) and the Hyades ( $625 \mathrm{Myr})$. Either the "dynamical evaporation" does not have (or has not yet had) the same effect on these three clusters, or they had different initial mass functions. 


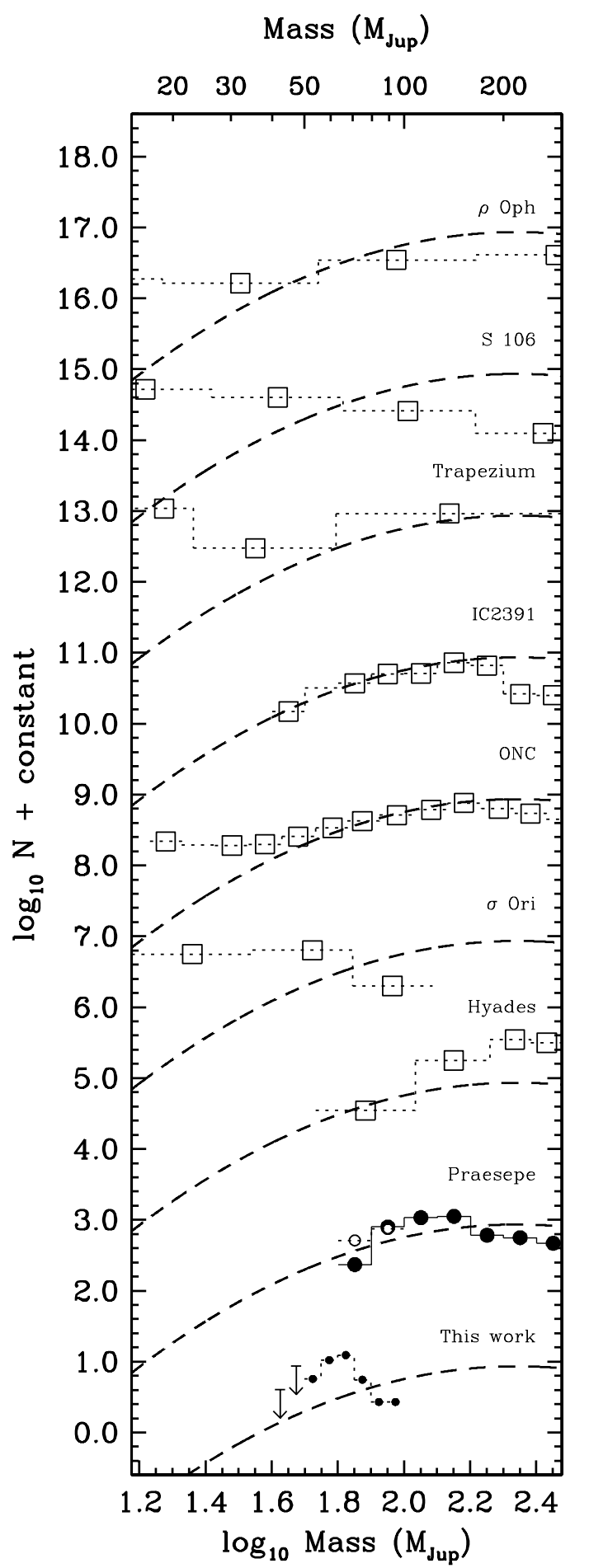

Fig. 10. Mass functions of various open clusters. From top to bottom: $\rho$ Oph ( 1 Myr, Marsh et al. 2010); S 106 ( 1 Myr, Oasa et al. 2006, their Fig. 10, middle panel for example); Trapezium ( 0.8 Myr, Muench et al. 2002); IC2391 ( 50 Myr, Boudreault \& Bailer-Jones 2009); ONC ( 5 Myr, Hillenbrand \& Carpenter 2000); $\sigma$ Ori ( 3 Myr, Bihain et al. 2009); Hyades ( 625 Myr, Bouvier et al. 2008); Praesepe ( 590 Myr, Boudreault et al. 2010 and from this work). We also show the lognormal fit to the Galactic field star MF from Chabrier (2003) as a dashed line. All the other MFs are normalized to this at the substellar limit $\left(\sim 72 M_{\text {Jup }}\right)$.

Another possibility is that Praesepe has a different binary fraction. Employing different cluster member selection criteria may also account for the observed MF discrepancies among clusters. Further studies are necessary to clarify these points.
The continuing rise of the MF into the substellar regime that we observe has also been observed in young clusters (as shown in Fig. 10), especially in $\sigma$ Orionis (Bihain et al. 2009), Trapezium (turn-over at $\sim 10-20 M_{\text {Jup }}$, Muench et al. 2002), $\rho$ Oph (MF rising to $\sim 10 M_{\text {Jup }}$, Marsh et al. 2010), and in the very low luminosity young cluster in S 106, where the MF increases or at least remains flat down to $\sim 10 M_{\text {Jup }}$ (Oasa et al. 2006). If we assumed a universal IMF, then it seems that the substellar MF of Praesepe has not evolved significantly since the cluster formed.

\section{Conclusions}

We have carried out the deepest survey to date of the old open cluster Praesepe, covering the central $0.59 \mathrm{deg}^{2}$ in the rizY bands. The survey probed a mass range from $\sim 100$ to $40 \mathrm{M}_{\text {Jup }}$ at $5 \sigma$ detection limit, with which we have derived the very low mass and substellar mass function of this cluster.

We compared our optical $i z$-bands data, combined with the $\Omega 2 \mathrm{k}$ NIR ( $J$ and $K_{\mathrm{s}}$ ) band observations from Boudreault et al. (2010), with theoretical loci of cluster members based on a dusty atmosphere (the AMES-Dusty model), to select cluster member candidates. Our final sample comprises 59 photometric candidates. We estimate that the contamination by field dwarfs is about $15 \%$, and that this does not affect the shape of MF. The contamination by galaxies and red giants is believed to be negligible. About two thirds of our cluster members have theoretical masses below the hydrogen-burning limit at $0.072 M_{\odot}$, and are therefore brown dwarf candidates. We emphasize that to claim cluster memberships for the candidates, follow-up astrometric or spectroscopic observations are required. However, given that the candidates are generally faint and these observations are very time-consuming, none of them has yet been confirmed in this way. The discussion in this contribution therefore refers to the mass function of photometric cluster member candidates.

The mass function we have inferred for the central $0.59 \mathrm{deg}^{2}$ of Praesepe is consistent with that inferred for a wider area by Boudreault et al. (2010) at a mass just below the substellar boundary, but deviates by $\sim 0.6 \mathrm{dex}$ in the next lowest mass bin, which may indicate that there is either a significant number of objects missing in the Boudreault et al. (2010) survey, or a higher concentration of substellar objects in the centre of Praesepe (as the Boudreault et al. survey is at a larger cluster radius). The latter possibility suggests that the dynamical evolution of very low mass stars is inefficient in this cluster, as proposed by Boudreault et al. (2010) for explaining the discrepancy between the Praesepe MF and Hyades MF.

The steady rise in the Praesepe MF down to $\sim 70 M_{\text {Jup }}$ and a turn-over there were unexpected for this old cluster. Such a significant peak has never been observed in any other cluster older than $50 \mathrm{Myr}$, but has been observed in several very young open clusters such as $\sigma$ Orionis or clusters in star-forming regions (e.g., Trapezium). This suggests that the dynamical interactions in Praesepe have very little effect on MFs, if we assume there is a universal initial MF.

Acknowledgements. This project was supported by DFGSonderforschungsbereich 881 "The Milky Way System". Some of the observations on which this work is based were obtained during LBT programme "LBT-F08-02". The LBT is an international collaboration among institutions in Germany, Italy and the United States. LBT Corporation partners are: LBT Beteiligungsgesellschaft, Germany, representing the Max-Planck Society, the Astrophysical Institute Potsdam, and Heidelberg University; Istituto Nazionale di Astrofisica, Italy; The University of Arizona on behalf of the Arizona university system; The Ohio State University, and The Research Corporation, on behalf of The University of Notre Dame, University of Minnesota and 
University of Virginia. Some data analysis in this article has made use of the freely available $\mathrm{R}$ statistical package, http://www.r-project.org. This research has made use of the SIMBAD database, operated at CDS, Strasbourg, France. This publication makes use of data products from the Two Micron All Sky Survey, Sloan Digital Sky Survey, and United Kingdom Infrared Telescope Infrared Deep Sky Survey.

\section{References}

Allard, F., Hauschildt, P. H., Alexander, D. R., et al. 2001, ApJ, 556, 357 Bailer-Jones, C. A. L., \& Mundt, R. 2001, A\&A, 367, 218

Bastian, N., Covey, K. R., \& Meyer, M. R. 2010, ARA\&A, 48, 339

Bihain, G., Rebolo, R., Zapatero Osorio, M. R., et al. 2009, A\&A, 506, 1169

Bouvier, J., Kendall, T. T., Meeus, G., et al. 2008, A\&A, 481, 661

Boudreault, S., \& Bailer-Jones, C. A. L. 2009, ApJ, 706, 1484

Boudreault, S., Bailer-Jones, C. A. L., Goldman, B., et al. 2010, A\&A, 510, A27

Caballero, J. A., Béjar, V. J. S., Rebolo, R., et al. 2007, A\&A, 470, 903

Caballero, J. A., Burgasser, A. J., \& Klement, R. 2008, A\&A, 488, 181

Chabrier, G. 2003, PASP, 115, 763

Chabrier, G., Baraffe, I., Allard, F., \& Hauschildt, P. 2000, ApJ, 542, 464

Chappelle, R. J., Pinfield, D. J., Steele, I. A., Dobbie, P. D., \& Magazzù, A. 2005, MNRAS, 361, 1323

Colina, L., Bohlin, R., \& Castelli, F. 1996, Instrument Science Report CAL/SCS, 8,1

Covey, K. R., Ivezić, Ž, Schlegel, D., et al. 2007, AJ, 134, 2398

Dahn, C. C., Harris, H. C., Verba, F. J., et al. 2002, AJ, 124, 1170
Fossati, L., Bagnulo, S., Landstreet, J., et al. 2008, A\&A, 483, 891 Friel, E. D., \& Boesgaard, A. M. 1992, ApJ, 387, 170

González-García, B. M., Zapatero Osorio, M. R., Béjar, V. J. S., et al. 2006, A\&A, 460, 799

Hambly, N. C., Steele, I. A., Hawkins, M. R. S., \& Jameson, R. F. 1995, Ap\&SS 109,29

Hauschildt, P. H., Allard, F., Ferguson, J., Baron, E., \& Alexander, D. R. 1999, ApJ, 525, 871

Hill, J. M., Green, R. F., \& Slagle, J. H. 2006, SPIE, 6267, 31

Hillenbrand, L. A., \& Carpenter, J. M. 2000, ApJ, 540, 236

Hodgkin, S. T., Pinfield, D. J., Jameson, R. F., et al. 1999, MNRAS, 310, 87

Lawrence, A., Warren, S. J., Almaini, O., et al. 2007, MNRAS, 379, 1599

van Leeuwen, F. 2009, A\&A, 497, 209

Magazzù, A., Rebolo, R., Zapatero Osorio, M. R., Martín, E. L., \& Hodgkin, S. T. 1998, ApJ, 497, L47

Marsh, K. A., Plavchan, P., Kirkpatrick, J. D., et al. 2010, ApJ, 719, 550

Muench, A. A., Lada E. A., Lada, C. J., \& Alves, J. 2002, ApJ, 573, 366

Oasa, Y., Tamura, M., Nakajima, Y., et al. 2006, AJ, 131, 1608

Pace, G., Pasquini, L., \& François, P. 2008, A\&A, 489, 403

Pinfield, D. J., Hodgkin, S. T., Jameson, R. F., et al. 1997, MNRAS, 287, 180

Schmidt, S. J., West, A. A., Hawley, S. L., \& Pineda, J. S. 2010, AJ, 139, 1808

Speziali, R., Di Paola, A., Giallongo, E., et al. 2008, Proc. SPIE, 7014, 158

Stern, D., Kirkpatrick, J. D., Allen, L. E., et al. 2007, ApJ, 663, 677

Taylor, B. J. 2006, AJ, 132, 2453

West, A. A., Hawley, S. L., Bochanski, J. J., et al. 2008, AJ, 135, 785

West, A. A., Morgan, D. P., Bochanski, J. J., et al. 2011, AJ, 141, 97

York, D. G., Adelman, J., Anderson, J. E., Jr., et al. 2000, AJ, 120, 1579 
W. Wang et al.: Mass function of Praesepe from deep LBT observations

Table 2. Journal of the LBT/LBC observations.

\begin{tabular}{|c|c|c|c|c|c|c|c|}
\hline Field & RA & Dec & Date & Filter & $\begin{array}{c}t_{\exp } \\
(\mathrm{min})\end{array}$ & $\begin{array}{l}\text { Seeing } \\
(\operatorname{arcsec})\end{array}$ & $\begin{array}{c}m_{5 \sigma} \\
(\mathrm{mag})\end{array}$ \\
\hline \multirow[t]{4}{*}{ PraeA } & $08: 40: 53.76$ & $+19: 52: 41.9$ & $2009-02-28$ & $r$ & 114 & 1.0 & 25.8 \\
\hline & & & $2009-02-28$ & $i$ & 42 & 1.0 & 25.8 \\
\hline & & & $2009-02-28$ & $z$ & 54 & 1.0 & 24.8 \\
\hline & & & 2009-02-28 & $Y$ & 18 & 1.0 & 21.3 \\
\hline \multirow[t]{4}{*}{ PraeB } & 08:39:14.23 & $+19: 52: 41.9$ & $2009-02-28$ & $r^{a}$ & 54 & 1.0 & 25.1 \\
\hline & & & 2008-03-06 & $i$ & 42 & 2.2 & 25.7 \\
\hline & & & $2009-02-28$ & $z$ & 54 & 1.0 & 25.2 \\
\hline & & & 2008-03-04 & $Y$ & 48 & 1.4 & 22.2 \\
\hline \multirow{4}{*}{ PraeC } & 08:39:14.36 & $+19: 27: 18.0$ & 2008-03-07 & $r$ & 90 & 1.9 & 25.6 \\
\hline & & & 2008-03-07 & $i$ & 30 & 2.0 & 25.8 \\
\hline & & & 2008-03-07 & $z$ & 30 & 1.0 & 25.1 \\
\hline & & & $2009-02-28$ & $Y$ & 18 & 1.0 & 21.2 \\
\hline \multirow[t]{4}{*}{ PraeD } & $08: 40: 53.63$ & $+19: 27: 18.0$ & $2008-12-29$ & $r$ & 99 & 1.0 & 25.8 \\
\hline & & & $2008-12-29$ & $i$ & 42 & 1.0 & 25.8 \\
\hline & & & $2008-12-29$ & $z$ & 39 & 1.0 & 24.8 \\
\hline & & & $2008-12-29$ & $Y$ & 18 & 1.0 & 21.9 \\
\hline
\end{tabular}

Notes. ${ }^{(a)}$ We also performed a shallower, 84-min pointing in $r$ on 2008-12-30. 
Table 3. The 22 low mass and 37 brown dwarf candidates in Praesepe.

\begin{tabular}{|c|c|c|c|c|c|c|c|c|c|c|c|c|c|}
\hline ID & RA(J2000) & Dec(J2000) & $\begin{array}{c}i \\
(\mathrm{mag})\end{array}$ & Err & $\begin{array}{c}z \\
(\mathrm{mag})\end{array}$ & Err & $\begin{array}{c}J \\
(\mathrm{mag})\end{array}$ & Err & $\begin{array}{c}K_{\mathrm{s}} \\
(\mathrm{mag})\end{array}$ & Err & $\begin{array}{l}T_{\text {eff }} \\
(\mathrm{K})\end{array}$ & $\begin{array}{c}M \\
\left(M_{\text {Jup }}\right)\end{array}$ & $\begin{array}{l}J_{\text {model }} \\
\text { (mag) }\end{array}$ \\
\hline A01 & $8: 40: 12.599$ & $19: 56: 50.33$ & 20.29 & 0.03 & 19.01 & 0.06 & 18.14 & 0.05 & 17.01 & 0.05 & 3023 & 109 & 16.39 \\
\hline $\mathrm{A} 02$ & $8: 40: 18.893$ & $19: 57: 07.40$ & 20.17 & 0.02 & 18.74 & 0.06 & 17.75 & 0.03 & 16.77 & 0.04 & 2729 & 93 & 16.90 \\
\hline A03 & $8: 40: 20.624$ & $19: 43: 40.72$ & 23.42 & 0.06 & 21.75 & 0.10 & 19.55 & 0.11 & 17.88 & 0.10 & 1921 & 58 & 19.04 \\
\hline A04 & $8: 40: 24.901$ & $19: 57: 15.52$ & 22.93 & 0.05 & 21.19 & 0.03 & 19.25 & 0.18 & 17.53 & 0.08 & 1940 & 59 & 18.99 \\
\hline A05 & $8: 40: 25.044$ & $19: 41: 44.56$ & 19.23 & 0.02 & 18.40 & 0.02 & 17.37 & 0.02 & 16.47 & 0.03 & 3093 & 113 & 16.27 \\
\hline A06 & $8: 40: 25.773$ & $19: 57: 08.28$ & 19.84 & 0.03 & 18.79 & 0.04 & 17.63 & 0.04 & 16.72 & 0.04 & 2821 & 98 & 16.74 \\
\hline A07 & $8: 40: 25.981$ & $19: 57: 07.02$ & 19.72 & 0.03 & 18.69 & 0.04 & 17.55 & 0.04 & 16.63 & 0.04 & 2900 & 102 & 16.61 \\
\hline A08 & $8: 40: 27.459$ & $19: 43: 19.69$ & 22.06 & 0.08 & 20.66 & 0.08 & 18.60 & 0.04 & 16.96 & 0.04 & 1997 & 61 & 18.82 \\
\hline A09 & .282 & 19 & 25.33 & 0.15 & 23.23 & 0.07 & 20.28 & 0.20 & 18.84 & 0.24 & 1855 & 56 & 19.22 \\
\hline A10 & 639 & $19: 5$ & 23.94 & 0.03 & 22.41 & 0.02 & 19.77 & 0.06 & 18.37 & 0.08 & 1902 & 57 & 19.09 \\
\hline A11 & 313 & .81 & 19.72 & 0.01 & 18.80 & 0.02 & 16.66 & 0.01 & 15.83 & 0.02 & 2351 & 75 & 17.73 \\
\hline A 12 & $8: 40:$ & 4.80 & 23.43 & 0.02 & 21.83 & 0.02 & 19.09 & 0.02 & 17.56 & 0.04 & 1842 & 55 & 19.26 \\
\hline A 13 & $8: 40: 49.194$ & $19: 5$ & 23.19 & 0.02 & 21.59 & 0.03 & 19.31 & 0.04 & 18.41 & 0.08 & 2040 & 62 & 18.68 \\
\hline A 14 & $8: 41: 08.239$ & $19: 47: 08.93$ & 23.13 & 0.09 & 21.55 & 0.04 & 19.05 & 0.02 & 17.69 & 0.03 & 1905 & 57 & 19.08 \\
\hline A 15 & $8: 41: 15.526$ & $19: 44: 11.43$ & 20.49 & 0.02 & 19.20 & 0.01 & 17.00 & 0.07 & 16.13 & 0.03 & 2169 & 67 & 18.25 \\
\hline A16 & $8: 41: 24.916$ & $19: 5$ & 19.11 & 0.01 & & 0.06 & & 0.01 & 15.25 & 0.01 & 2356 & 75 & 17.72 \\
\hline A 17 & $8: 41: 26.483$ & $19: 51: 59.73$ & 21.38 & 0.01 & & 0.02 & & 0.02 & 17.34 & 0.02 & 2294 & 72 & 17.86 \\
\hline B01 & $8: 38: 39.176$ & $19: 42: 54.85$ & 20.21 & 0.01 & 18.99 & 0.01 & 17.30 & 0.03 & 16.26 & 0.02 & 2373 & 76 & 17.68 \\
\hline B02 & $8: 38: 59.208$ & $20: 02: 32.63$ & 22.99 & 0.03 & 21.31 & 0.01 & 19.29 & 0.12 & 17.95 & 0.10 & 2050 & 63 & 18.65 \\
\hline B03 & 8:39:06.931 & $19: 51: 00.19$ & 19.28 & 0.01 & 18.26 & 0.09 & 16.98 & 0.01 & 16.13 & 0.02 & 2712 & 92 & 16.93 \\
\hline B04 & .285 & 63 & 62 & 0.01 & & 0.09 & 17.64 & 0.04 & 16.66 & 0.03 & 3093 & 113 & 16.27 \\
\hline B05 & 75 & & & 0.05 & & 0.03 & & 0.07 & 17.77 & 0.09 & 2060 & 63 & 18.61 \\
\hline B06 & 10 & 06 & & 0.02 & & 0.03 & & 0.05 & 16.68 & 0.03 & 1892 & $F$ & 19.12 \\
\hline B07 & & & & 0.01 & & 0.01 & 4 & 0.02 & 16.06 & 0.02 & 2674 & 9 & 17.00 \\
\hline B08 & 841 & & 3 & 0.02 & 2 & 0.03 & & 0.05 & 16.84 & 0.03 & 1867 & & 19.19 \\
\hline B09 & & & 6 & 0.03 & & 0.04 & 19 & 0.09 & 17.95 & 0.11 & 2112 & 6 & 18.44 \\
\hline $\mathrm{C} 01$ & 01 & & .27 & 0.01 & & 0.01 & 16 . & 0.09 & 16.13 & 0.02 & 2590 & 证 & 17.18 \\
\hline $\mathrm{C} 02$ & & & & 0.06 & & 0.02 & & 0.10 & 18.14 & 0.12 & 21 & & 18.21 \\
\hline $\mathrm{C} 03$ & 26 & & & 0.03 & & 0.02 & & 0.06 & 17.34 & 0.05 & 1808 & 5 & 9.49 \\
\hline $\mathrm{C} 04$ & 9.835 & & & 0.01 & & 0.01 & & 0.04 & & 0.07 & 2310 & 7 & 17.83 \\
\hline $\mathrm{C} 05$ & $8: 39: 00.756$ & 38.70 & 19.08 & 0.01 & 18 & 0.01 & 16.70 & 0.01 & 15.77 & 0.01 & 2626 & 88 & 17.10 \\
\hline C06 & $8: 39: 02.279$ & $19: 37: 38.47$ & 22.32 & 0.01 & 20.85 & 0.01 & 18.72 & 0.07 & 17.88 & 0.08 & 2159 & 67 & 18.29 \\
\hline $\mathrm{C} 07$ & $8: 39: 11.773$ & 5.63 & 21.53 & 0.01 & 20.27 & 0.01 & 18.61 & 0.10 & 17.44 & 0.06 & 2381 & 76 & 17.66 \\
\hline $\mathrm{C} 08$ & $8: 39: 22.309$ & 7.41 & 22.06 & 0.01 & 5 & 0.01 & 18.42 & 0.10 & 16.96 & 0.04 & 2062 & 63 & 18.61 \\
\hline $\mathrm{C} 09$ & & & & 0.01 & & 0.01 & & 0.10 & 16.41 & 0.03 & 2160 & 6 & 18.28 \\
\hline $\mathrm{C} 10$ & 196 & & 21 & 0.01 & & 0.01 & & 0.01 & & 0.02 & 2462 & 7 & 17.47 \\
\hline $\mathrm{C} 11$ & & & & 0.12 & & 0.03 & & 0.18 & 18.43 & 0.13 & 1786 & - & 19.64 \\
\hline $\mathrm{C} 12$ & & & & 0.01 & & 0.01 & & 0.01 & & 0.01 & & 6 & 18.09 \\
\hline $\mathrm{C} 13$ & & & 6 & 0.03 & & 0.01 & 6 & 0.07 & 18.16 & 0.11 & 2007 & 6 & 18.79 \\
\hline D01 & & & 9 & 0.01 & & 0.02 & 8 & 0.01 & 16.25 & 0.02 & 3 & 9 & 16.98 \\
\hline D02 & & & .64 & 0.07 & & 0.04 & 4 & 0.19 & 18.06 & 0.11 & 1703 & 5 & 20.21 \\
\hline D03 & 29 & & .33 & 0.05 & & 0.05 & 5 & 0.24 & 18.65 & 0.20 & 1896 & 5 & 19.11 \\
\hline D04 & $8: 40:$ & 6.21 & 24. & 0.14 & & 0.07 & 1 & 0.17 & 18.10 & 0.12 & 1848 & 5 & 19.24 \\
\hline D05 & $8: 40:$ & 1.23 & & 0.15 & & 0.06 & & 0.12 & 17.40 & 0.06 & 1760 & 52 & 19.81 \\
\hline D06 & $8: 40:$ & & & 0.01 & & 0.04 & & 0.01 & 16.10 & 0.02 & & 81 & 17.37 \\
\hline D07 & $8: 40: 2$ & 1.58 & 23.70 & 0.08 & 2 & 0.05 & 19.71 & 0.13 & 18.44 & 0.16 & 1947 & 59 & 18.97 \\
\hline D08 & $8: 40: 3$ & 9.49 & 22.04 & 0.02 & & 0.01 & 18.07 & 0.02 & 16.76 & 0.02 & 1962 & 59 & 18.93 \\
\hline D09 & $8: 40: 43.705$ & & 25.40 & 0.17 & 23.39 & 0.10 & 21.10 & 0.38 & 18.66 & 0.14 & 1705 & 50 & 20.19 \\
\hline D10 & & & & 0.05 & & 0.04 & & 0.05 & 17.20 & 0.04 & & 57 & 19.11 \\
\hline D11 & & & & 0.03 & & 0.03 & & 0.04 & 17.10 & 0.03 & 1847 & 55 & 19.25 \\
\hline D12 & & & & 0.02 & & 0.03 & & 0.05 & 15.61 & 0.02 & 2320 & 73 & 17.81 \\
\hline D13 & & & & & & 0.06 & & 0.12 & & 0.06 & & & 20.39 \\
\hline D14 & & & 23.01 & 0.0 & 21.23 & 0.06 & 19.08 & 0.06 & 17.81 & 0.07 & 2015 & & 18.76 \\
\hline D15 & & & 23.66 & 0.04 & & 0.04 & 20.22 & 0.16 & 19.35 & 0.25 & 2216 & 69 & 18.10 \\
\hline D16 & & & 24.41 & 0.0 & 22.88 & 0.06 & & 0.17 & 19.41 & 0.30 & 1903 & 57 & 19.09 \\
\hline D18 & $1: 15.768$ & & 19.79 & 0. & & 0.02 & 17.35 & 0.01 & & 0.02 & 2588 & 8 & 17.19 \\
\hline D1 & & & & 0.02 & & 0.02 & & 0.08 & & 0.12 & & 68 & 18.20 \\
\hline D20 & & & & 0.03 & 22 & 0.04 & & 0.09 & 17.86 & 0.07 & 1807 & 54 & 19.49 \\
\hline D21 & $8: 41: 36.404$ & $19: 25: 06.45$ & 20.71 & 0.01 & 19.60 & 0.01 & 18.01 & 0.03 & 17.24 & 0.05 & 2513 & 82 & 17.35 \\
\hline
\end{tabular}

